\title{
陆地生态系统碳密度格局研究概述
}

\author{
吕超群 孙书存
}

(南京大学生物系, 南京 210093)

\begin{abstract}
摘 要 准确了解陆地生态系统中碳密度的时空格局及其影响因子和作用机制, 对于估算和预测不同类型生态系 统中的植被和土壤的碳存储能力、判定碳汇、制定缓解全球变化的合理政策措施, 具有重要意义。该文综述了现有 研究中发现的世界陆地生态系统碳密度空间分布的地带性规律及中国陆地生态系统碳密度格局的独特特点。在 全球尺度上 植被碳密度分布与植物生物量格局基本一致, 除北方森林外其余大部分随纬度升高而减小; 圷壤碳密 度则随纬度升高而增大。陆地生态系统中北方森林和热带森林的总体碳密度最高, 不同的是, 前者的碳主要集中 在土壤中, 而后者则集中在植被中。但在区域尺度上,由于气候、地形及人类活动影响, 这种规律性可能会发生变 化甚至不起作用。水热条件、土壤养分、生物多样性、气候和大气 $\mathrm{CO}_{2}$ 浓度的变化以及土地利用与覆盖变化等是碳 密度空间格局形成和发生变化的驱动因子。在某一特定区域，它们通过直接或间接提高植被净初级生产力，抑制 呼吸和分解作用来增加陆地生态系统碳密度。综合分析特定时空条件下各因子对碳存储量的影响是解释碳密度 分布现状, 预测碳密度格局变化的关键, 但目前的研究对各项驱动因子的作用机制、影响强度及多个因子间的相互 作用仍不是很清楚, 需要加强该方面的研究力度。碳密度研究中的数据获取、机理分析和过程模拟等方面仍存在 很大的不确定性, 因此有必要建立规范统一的碳密度测量估算系统和更为精准有效的估算模型, 进行多尺度、多精 度水平的综合研究。
\end{abstract}

关键词 碳密度 陆地生态系统 空间格局 影响因子

\section{A REVIEW ON THE DISTRIBUTION PATTERNS OF CARBON DENSITY IN TERRESTRIAL ECOSYSTEMS}

\author{
LÜ Chao-Qun and SUN Shu-Cun \\ (Department of Biology , Nanjing University , Nanjing 210093, China)
}

\begin{abstract}
Terrestrial ecosystems are a large carbon density and play an important role in the global carbon budget and mitigating global warming by carbon sequestration. To encourage additional carbon sequestration and storage in global vegetation and soils, we need to be clear about the distribution patterns of carbon density and the factors that influence these patterns. Therefore, the characteristics of the distribution patterns of carbon density in terrestrial ecosystems of the world and China are reviewed in this paper.

At the global scale, the distribution of carbon in vegetation corresponds with the spatial pattern of biomass and generally decreases from low-latitude to high-latitude with the exception of boreal forests. In contrast, soil organic pools of carbon increase along the same gradient. The largest stores of carbon in biomass are in tropical and boreal forests, and the largest stores of soil carbon are in the high latitude ecosystems (boreal forest and tundra). Total carbon density of both vegetation and soils are highest in tropical and boreal forests. In the tropics more carbon is stored in vegetation than in soils, while in the boreal region far more carbon is stored in the soils. At the regional scale, these patterns might vary due to differences in climate, topography and human influence among regions.

Several major factors, including climatic conditions , soil nutrients, biodiversity , climate and atmospheric $\mathrm{CO}_{2}$ changes, land use and cover changes, all contribute to the storage and maintenance of carbon. For example , carbon density will be high in regions where temperature and precipitation are favorable for abundant plant growth. The enhanced carbon sequestered in response to elevated levels of $\mathrm{CO}_{2}$ or nitrogen , or their combination, is less in species-poor than in species-rich regions. In a word, they can raise the carbon density in terrestrial ecosystems by directly or indirectly accelerating net primary production , or constraining respiration and decomposition. However, in spite of its great significance for explaining the present distribution patterns of carbon pools and estimating future changes, the mechanisms by which these processes occur are not fully under-
\end{abstract}


stood. It is critical that we strengthen our research effort in this area of study.

Although a great deal of effort has been put into the carbon density researches, there still remains much uncertainty regarding data collection, mechanistic explanations, and model construction in the relevant studies. In the future, we should establish a standardized and unified system to estimate carbon density , produce more accurate and useful models, and perform integrated studies at multi-scales and multi-resolutions levels.

Key words Carbon pools , Terrestrial ecosystems , Distribution patterns , Influence factors

$\mathrm{CO}_{2}$ 和其它温室气体向大气中的排放量增加， 已经引起过去 100 年里全球平均气温升高, 气候异 常;持续温室气体累积将会导致生物地带重新分配， 海平面升高，降水方式改变和其它一系列全球变化 的发生 (Donald et al. , 1997)。将人为排放的碳转入 陆地生态系统, 降低温室气体排放量, 可以部分减缓 全球变化的进程 (Emily et al , , 2000 ; Robin et al. , 2000)。

陆地生态系统的碳储量是研究陆地生态系统与 大气碳交换的基本参数, 也是估算陆地生态系统吸 收和排放含碳气体数量的关键要素 (Valentini, 2000)。准确了解当前各种生态系统碳储库的大小、 位置, 碳排放和碳吸收通量, 并切实评估不同类型植 被和土壤的碳存储能力, 是制定合理政策措施, 提高 世界植被和土壤的碳吸收速度, 增加陆地碳存储量 的基础（Robin et al，2000)。同时 模拟和估算长时 间、大尺度上的区域碳储量, 对于了解世界主要陆地 生态系统碳存储的价值, 并进一步制定公平合理的 碳吸收补偿政策有重要意义( Suzi et al., 2001)。 到目前为止, 已经开展了许多有关陆地生态系统碳 密度格局的研究, 包括全球水平和区域水平的研究 工作(Schimel，1995)，但这些研究的结果各异，特别 是国家或地区等区域尺度上的碳储量分布测算，由 于各项研究对陆地生态系统的分类或者某类生态系 统内部的划分不能完全统一,采用的量测方法和基 础数据也不尽相同, 所以其结论常不能一致, 即使是 对同一地区的碳储量进行估测，结果也会存在很大 差异(方精云等 ,1996b; $\mathrm{Ni}, 2001$ )。在对影响碳密度 分布的因子及其机制的研究中, 也存在着争论, 选用 不同的样地或物种, 以及实验方法的差异都可能忽 略或屏蔽某一因子的作用, 或者过分夸大碳存储对 某一因子的依赖, 导致机理分析的结论不能广泛有 效地解释实际现象 (Eric et al., 2000 ; Christian \& Michael, 2000)。另外, 各种描述陆地碳循环及碳储 量的模型, 在建模机理、参数选用和过程模拟等方面 都存在很大差异 (Cramer et al . , 1999)，因而不同模 型的适用范围及有效性都有所不同, 特定地区模型 模拟的完善仍需要结合模型对比研究和其它的技术
手段。

针对碳密度估算中存在的问题 现在的碳储量 及碳循环研究开始重视地区间的关联性和整体性 (Valentini，2000)。并将 3S 技术等更为直观、动态的 监测分析手段应用到碳密度估算与建模中，观测陆 地生态系统碳密度的时空变异, 增强碳密度研究的 可比性(王绍强等, 1999; 方精云等 ,1996a; Cramer et al . , 1999)。陆地生态系统碳存储量对全球气候变 化和大气 $\mathrm{CO}_{2}$ 浓度升高的响应也成为当前碳密度研 究的重点(King et al. , 1997)。

本文综述了目前在全球陆地生态系统碳密度格 局研究中得到的地带性规律和中国陆地生态系统碳 密度分布的特点, 阐述了影响碳密度分布的主要驱 动因子及其作用机制, 旨在突出陆地生态系统碳密 度研究的重要性, 总结碳密度研究的现状, 揭示其中 存在的制约因素和不确定性，以提高碳密度估算的 准确性和预测的可靠性, 使碳密度研究能够更好地 为政策措施的制定提供依据。

\section{1 全球陆地生态系统碳密度分布格局}

通过 $\mathrm{CO}_{2}$ 的光合固定和呼吸释放过程, 碳在海 洋、大气和陆地生物圈 (包括植物和土壤) 这 3 个主 要的地球碳储库间流动 (Emily et al. ,2000)。近年 来研究发现, 尽管化石燃料燃烧的碳释放量大幅度 增加, 但大气和海洋的年净吸收量都增加很少, 这部 分额外碳沉降在很大程度上是由陆地生产力的提高 来实现的 (Fan et al ， 1998)。所以，准确估算陆地 生态系统碳存储的现状, 对于研究全球碳通量及气 候变化有重要意义(Donald et al . , 1997)。世界资源 研究所 (World Resource Institute, WRI) 在 2000 年所 做的全球生态系统示范分析 (Pilot Analysis of Global Ecosystem，PAGE)中，对已有的陆地生态系统碳密度 估算结果进行整理、校正 将其和近期土地利用覆盖 变化特征数据结合起来, 进而分析全球陆地生态系 统碳密度空间格局的现状。

\section{1 全球陆地生态系统植被碳密度分布格局}

碳吸收 (Carbon sequestration) 是指随着植物生长 及其生物量的增加，植物从空气中吸收大量碳并将 
其储存在植物组织中的过程; 尽管在植物、土壤、大 气中存在着连续的碳交换, 但仍有大量的碳储存在 了植物叶片、木质部分和土壤养分中, 这个碳量被称 作碳存储量 (Carbon storage)。碳吸收速率和碳存储 量的增加可以减慢大气中的 $\mathrm{CO}_{2}$ 累积速度, 缓解全 球升温(Robin et al. ,2000)。地球上的植被每年吸 收的 $\mathrm{CO}_{2}$ 约折合 $120 \mathrm{Pg} \mathrm{C}\left(1 \mathrm{Pg}=10^{15} \mathrm{~g}\right)$, 以植物总 初级生产力的形式表现。碳存储量就是从吸收的碳 量中去掉植物呼吸、土壤微生物分解和人为干扰引 起的碳损失所得的, 大约为 $0.7 \mathrm{Pg} \mathrm{C} \mathrm{a}^{-1}$ (Emily et $a l$. ,2000)。碳密度 (Carbon density) 是指单位面积上 的碳存储量, 是生态系统碳存储能力的重要指标之

由于取样地的自然地理状况存在很大差异，不 同项目的研究目的和方法也不相同, 而且对于植被 和土壤类型的界定不很明确, 所以已有的碳密度研 究结果存在着相当大的差异 (Thomas et al ., 1988)。 Olson 等(1983) 将世界主要生态系统按照其植被含 碳量分为 44 类, 以 $0.5^{\circ} \times 0.5^{\circ}$ 经纬格为一个空间单 元 运用遥感技术和野外考察获取的样地生物量数 据，估算出各类生态系统的植被生物量，然后通过特 定的生物量/碳量转换率, 来估计它们的最大、最小 和中等碳密度。Olson 的碳估测值一度被认为是 最 普遍被采用的, 在全球尺度上对生物量及碳密度空 间分布的清楚描述” (Gaston et al. , 1998) ,但 Olson 的植被分类系统是对全球植被类型的概括和简化， 忽略了大量植被类型细节和地区间的组成差异性， 所以 Olson 的植被碳存储量估测结果目前已几乎被 近期的国家或地区尺度上的研究所取代。尽管如 此,PAGE 的研究人员仍将其作为全球尺度上植被 碳密度估算中的唯一一个具一致性的数据系列来使 用, 他们把 Olson 的植被碳储量估测结果与 IGBP 定 义的生态系统分类结合起来, 利用一张建立在近期 全球土地利用特征数据(Loveland et al ，2000)基础 上的植被图，对全球陆地生态系统植被碳密度空间 格局做了重新估算。得到的植被碳密度是以 Olson 的最大碳密度估测值为依据的, 全球陆地生态系统 植被碳存储量可达 268 901 Pg(表 1)。最大碳密度 存在于热带森林中, 接下来依次是北方森林、温带森 林和热带稀树草原。非木本的草地储存的碳少于森 林, 植被稀疏或裸露的荒漠地区碳存储能力最小, 实 际上这也是与植物生物量分布相一致的。在森林生 态系统中, 从热带阔叶林、亚热带常绿阔叶林、落叶 阔叶林及灌丛、亚热带热带山地针叶林、亚热带热带
针叶林、温带落叶阔叶林及灌丛, 碳密度是随纬度的 增加而降低的; 草地和农业用地的植被碳储量也基 本随纬度升高而降低 (表 1 )。在局部地区, 碳密度 的分布还表现出经度地带性规律, 例如在中国北部 随经度减小碳密度呈递减趋势 (王绍强等, 1999)。 1996 年到 1998 年间对 15 个欧洲森林生态系统的净 碳交换 (NEE) 研究也表明, 靠海的生态系统可以比 相同纬度上趋大陆性的系统吸收更多的碳 (Valentini , 2000)。

\section{2 全球陆地生态系统土壤碳密度分布格局}

土壤碳库存储量是陆地三大碳库中最大的, 但 其不确定性也最大。目前国内外有关土壤有机碳蓄 积量的研究一般采用土壤类型法、植被类型法、生命 地带类型法、模型法和相关关系统计法等方法 (王绍 强等 2001)。土壤中的有机碳以死的植物组织和土 壤微生物的形式存在。从土表向下的 $100 \mathrm{~cm}$ 土层 是最能与大气发生直接相互作用, 对土地利用和环 境变化最敏感的部分 (Batjes, 1996) ,多数学者以世 界土壤图等资料为主要依据，用土壤类型法对 $1 \mathrm{~m}$ 深度内的碳库存储量做了估算, 但事实上, 有一些土 壤的深度低于 $1 \mathrm{~m}$,一些大于 $1 \mathrm{~m}$, 计算过程中需要 做相应的调整。 20 世纪 70 年代,Bohn 利用土壤分 布图及相关土组 (Soil association) 的有机碳含量, 通 过土组合并 相近土类间数据的相互引用及插值, 计 算出全球有机碳库为 $2.95 \times 10^{18} \mathrm{~g}$ (Bohn ,1976)。20 世纪 80 年代以来，许多科学家对不同尺度上的土壤 碳蓄积量进行了估算, 但由于数据来源和计算方法 的不同，所得的结果差异很大(Post et al. , 1982)。 Batjes (1996) 分析了 4000 多个土壤剖面数据, 计算 出世界粮农组织 ( Food and agriculture organization, FAO) 定义的每一种土壤类型在几个深度间隔上的 平均土壤有机碳 (SOC) 含量, 他将 FAO-UNESCO 的 世界土壤电子地图分为 $30 \times 30$ 的棚格，根据每一柾 格中各土壤类型的比例给各土壤有机碳含量赋予权 重, 从而计算出有机碳的总量, 他的结果是土壤上层 $100 \mathrm{~cm}$ 的有机碳含量在 $1462 \sim 1548 \mathrm{Pg}$ 之间( Batjes，1996)。PAGE 的研究人员用最新世界土壤地图 的 $5 \times 5$ 栅格和 Batjes 得出的平均有机碳 (SOC) 含量 重复了 Batjes 的实验, 估测出土壤上层 $100 \mathrm{~cm}$ 的有 机碳含量为 $1484 \mathrm{Pg}$, 在此基础上分析全球土壤有 机碳的空间分布。最大土壤碳密度出现于高纬度地 区 (如北方森林和冻原) ,北方地区土壤碳密度最高 可达 $1250 \mathrm{Mg} \mathrm{hm}^{-2}$ (表 1) 热带森林、热带稀树草 原和温带草原也是重要的土壤碳储库。 


\section{3 全球陆地生态系统碳密度总体空间格局}

应用 GIS 技术, 以 Olson 的最高植被碳密度估算 值和 WRI 的土壤碳密度估测为基础，将上面工作中 得到的两幅图进行叠加，可以清晰体现出全球碳存 储的位置和大小, 这种方法测得的全球碳储量为 $1752 \sim 2385 \mathrm{Pg}$ (表 1) , 最大和最小估测值之间的巨 大差距说明我们对碳密度的估算仍存在很大的不确 定性。WRI 数据表明森林占陆地生态系统碳存储量 的 $40 \%$,草地占 $34 \%$, 农田占 $17 \%$ (Emily et al.， 2000 ; Robin et al . 2000)。其中最高碳密度存在于 北方森林和热带森林中, 但碳在这二者中聚集的地 方不同, 热带植被中的碳密度远高于土壤中的, 而在 北方森林区更多的碳存在于土壤中 (表 1)。北方泥 炭地因其极高的土壤碳密度而具有很大的重要性。 草地碳密度一般小于森林, 但因其幅员广阔, 总碳量 远高于温带森林, 甚至与热带森林相当 (Robin et al. 2000)。

为了更清楚地表现森林生态系统的巨大碳储 量,PAGE 研究组将陆地生态系统划分为 3 个纬度 带, 低纬度 $\left(25^{\circ} \mathrm{S} \sim 25^{\circ} \mathrm{N}\right)$ 对应热带亚热带生态系 统, 中纬度 $\left(25^{\circ} \sim 50^{\circ} \mathrm{N}\right.$ 和 $\left.25^{\circ} \sim 50^{\circ} \mathrm{S}\right)$ 对应温带生态 系统, 高纬度 $\left(50^{\circ} \sim 90^{\circ} \mathrm{N}\right.$ 和 $\left.50^{\circ} \sim 90^{\circ} \mathrm{S}\right)$ 主要为北方 森林和苔原, 其中北纬 50 以北温暖的西欧国家也被 划入中纬度范围。采用 WRI 结合近期土地覆盖数 据对 Olson 的生态系统组进行调整后所得到的生态 系统类型组, 估算了不同类型陆地生态系统中植被 及土壤的具体碳存储量和碳密度数据随纬度的变化 (表 1)。

\section{4 中国自然植被及土壤的碳密度格局}

中国陆地生态系统的碳密度分布状况与全球陆 地生态系统碳密度空间分布的规律在大体上是吻合 的，这表现在中国东部地区，由热带雨林向北到北方 针叶林之前植被碳密度基本随纬度升高而降低, 土 壤碳密度随纬度升高而增加。但由于气温、降水、季 风和地形等区域条件的影响, 中国陆地生态系统的 碳密度格局又有其自身的独特特点。

中国自然植被碳密度格局与我国植被生物量的 空间分布基本是一致的(王效科等,2001)。王绍强 等 (1999b) 就不同类型植被碳密度分布的研究中指 出, 在东部地区随热量的递减由南向北, 植被类型从 热带雨林、季雨林、常绿阔叶林、落叶与常绿阔叶混 交林、落叶阔叶林, 到针阔混交林, 植被碳密度是逐 渐减少的, 总体上表现出东部地区植被碳密度随纬 度增加而降低的趋势, 但东北地区的寒温带、温带山
地针叶林植被碳密度很高。在北部地区, 随水分的 递减由东向西,从湿润温带森林, 向西到松嫩草原、 内蒙古草原, 以至草被稀疏矮小的干草原和干旱少 雨的漠境, 植被覆盖率递减, 植被碳密度也是逐渐降 低的, 即我国北部地区植被碳密度具有随经度减小 而递减的趋势。中国植被碳密度水平分布并非简单 地只按上述经线与纬线作东西或南北向排列，由于 大地形关系和我国季风特征的综合作用碳密度分布 还表现出一定的偏转,在我国中西部之间具有一条 过渡带, 与腾冲-黑河人口分界线平行, 植被碳密度 在界线两旁差异是比较大的(王绍强等, 1999b)。

王效科等(2001) 以省份区划为基础，对中国森 林生态系统碳密度空间分布进行研究, 发现我国森 林植物碳密度较高的省份为黑龙江、吉林、西藏和海 南，小于 $53.1 \mathrm{mg} \mathrm{hm}$, 尽管西藏的森林面积很小， 但现存森林的植物碳密度很高, 如西藏的雅鲁藏布 江的大拐弯处是我国目前森林生物量最高的地方； 植物碳密度较小的省包括广东、广西、湖北、湖南、江 西、浙江、江苏、安徽和山东, 小于 $12.4 \mathrm{mg} \mathrm{hm}$ 。 由此指出中国森林的植被碳密度有从东南向东北和 西增加的趋势, 并与人口密度负对数显著相关(王效 科等 ,2001) 这与王绍强等 (1999b) 运用植被类型法 研究获取的结论存在很大差异。对于中国草地碳存 储量的研究相对较少, 但结论同样存在争议, 其中一 致认同的是 草地的植被碳密度中沼泽最高, 其次为 热带灌丛、稀树草原和温带草甸草原及暖温带灌丛， 高山荒漠和温带荒漠最低(方精云等,1996a；倪健， 2001)。

中国土壤是一个巨大的碳库, 在全球碳循环及 全球气候变化中起着相当重要的作用 (王绍强等, 1999a；1999b)。中国土壤有机碳储量的空间分布总 体上表现出:在东部地区大致随纬度的增加而递增， 在北部地区随经度减小而递减的趋势; 但存在一定 的偏转,中西部地区的土壤碳密度差异较大 ; 在西部 地区则呈现随纬度减小而增加的趋势 (王绍强等, 2000)。

\section{2 陆地生态系统碳密度的影响因子}

陆地生态系统的碳存储能力受多个因子及其相 互作用的影响。就植被来说, 其碳存储量代表了碳 获得过程 (光合作用, 树木生长, 树龄增长) 与碳释放 过程(生物呼吸 树木死亡)之间的平衡状况，土壤碳 存储量主要取决于植被凋落物向土壤的归还量和碳 在土壤中的积累, 以及调落物的微生物分解和土壤 
碳的氧化、降解及扰动 (Fang et al. , 1998 ; Fan et $a l$. , 1998)。影响其中任何一个过程的某个因子发 生变化都会引起生态系统碳储量的波动, 这类因子 即为引起碳密度格局发生变化的驱动因子。单位面 积上的碳存储量 (即碳密度) 对各因子的响应取决于 区域范围内碳输入和碳支出对所发生变化的敏感性 比较和受影响程度比较, 简言之, 在某一特定区域 内 提高净初级生产量的变化往往会促进生态系统 碳存储量的增加, 而有利于呼吸或分解过程的变化 则可能降低碳存储量(King et al . , 1997)。

\section{1 温度与降水}

从植物净初级生产量中去除被动物取食的部分 和凋落物产量, 即为以植物组织形式表现的生物量 增量。某一特定时刻,生态系统单位面积上积存的 生活有机质就是生物量。可见, 生物量实际上是净 生产量的累积量(张金屯等,2003)。由于温度和降 水在很大程度上影响着植物生产力和生物量的大 小, 而单位面积上的植物生物量与植被碳密度显著 相关，所以温度和降水是限制陆地生态系统植被碳 密度分布格局的重要因子。在水热因子的组合有利 于植物生长的地区 植物生物量大 植被碳密度也较 高, 如热带森林植被是陆地生态系统的一个极为重 要的碳储库, 其碳存储量最高可达 $256 \mathrm{Pg}$, 这是因为 热带地区终年高温多雨, 水分、热量条件充足, 植物 生长旺盛, 光合作用效率高, 种类组成丰富, 群落结 构复杂, 生物量高(FAO/UNEP，1981)。群落的光合 作用效率越高, 意味着净初级生产量占总初级生产 量的比例越大, 年净初级生产力的差异在一定程度 上可以反映不同植物群落类型光合固碳能力的差别 (许大全 2002)。但植物生物量和碳密度的大小是 由光照、温度、降水和土壤养分等多个因子共同决定 的，各因子的影响程度由于植物物种和其生长阶段 的不同也有差异, 所以不能说哪一个因子是植被碳 密度的决定因子，综合考虑上述因素可部分地解释 不同气候区以及不同生态系统类型间的碳密度差 异。群落组成和林龄长短对植物生物量积累也有很 大影响，如北方寒温带、温带山地针叶林大多为原生 林, 植物生长茂密, 种群密度大, 生物量相对比较大, 所以寒温带、温带山地针叶林的植被碳密度也比较 高, 再加上其土壤含碳量是低纬度森林区的两倍还 多 (Emily et al . ,2000) 故北方森林生态系统的总体 碳密度(包括植被和土壤)高于热带森林 (表 1)。

土壤有机碳库的动态变化主要取决于植物凋落 物的归还量和枯枝落叶的分解速率, 以及土壤生物
的呼吸速率和土壤表层的流失量(刘国华等，2003)。 气候不仅影响植物生产力和碳向土壤的输入, 而且 还会影响土壤碳的积累( Eric et al. ,2000)。Post 等 (1982)对土壤碳的研究指出, 土壤碳密度一般随降 水量的增加而增加, 但在特定降水量水平上, 它又随 温度升高而降低。温度对土壤碳含量的影响, 存在 着两种争论，一种说法认为, 潮湿、寒冷土壤的贫氧 化作用会阻止分解, 升温可以提高土壤有机质 (SOM)的分解速率, 因而随纬度升高, 年平均温度 (Mean annual temperature, MAT) 降低, 碳周转时间 ( Turnover time, TT) 延长, 故高纬度低温地区生长的 北方针叶林和苔原的土壤碳含量最高 (Eric et al., 2000)。另一种说法认为, 只有一小部分碳对于温度 变化敏感, 土壤碳分解速率的变化与温度无关, 调落 物生产力随年平均温度的升高而降低，即碳输入的 减小, 而非分解速率的提高, 才是土壤碳含量随温度 升高而减少的真正原因 (Christian \& Michael，2000)。 Post 等(1997)的模拟研究表明,1910 1975 年间全球 土壤有机碳含量呈减少趋势, 从 1975 年以后, 土壤碳 的增加导致整个模拟过程中出现了一个约 $13.6 \mathrm{Pg}$ $(1 \%)$ 碳的净汇。而土壤碳的损失正与凋落物输入的 减少相对应、随凋落物归还量增加，土壤碳损失减慢， 甚至出现扭转。红壤有机质分解特征研究也证实, 调 落物向土壤归还量的减少是部分地区土壤碳密度相 对较小的主要原因。李忠佩等(2002)发现红壤地区 的土壤质地粘重 酸度较高, 这种环境不利于有机质 快速分解, 因此他们指出旱地红壤长期以来保持较低 的碳含量, 并不是因为土壤有机质的分解速率快, 而 主要是因为侵蚀严重和有机物质的进入量低所致。

水热因子间的相互作用对碳密度空间格局也有 很大影响, 例如温度改变会影响蒸腾作用, 引起土壤 水分含量的变化，从而改变土壤有机质的分解速率， 所以温度不应作为单独的影响因子来考虑 (Donald et al.,1997)。预计在本世纪末全球将变暖 4 10 ${ }^{\circ} \mathrm{C}$, 这会提高分解速率、养分释放速率和初级生产 力, 这种情况下碳平衡的净效应主要取决于土壤湿 度 (James et al 。,2001)。土壤湿度是降水和蒸发蒸 腾作用相平衡的结果。降水通过影响植物光合作用 的水分需求、水分平衡以及碳固定量来影响植被的 地带分布和净初级生产力 (Prentice \& Fung, 1990)。 水分缺失可以使光合作用和植物生长急剧减小, 甚 至停止, 但水分过多和温度降低一样会抑制土壤呼 吸, 影响植物的正常生长。在较干旱地区, 降水是净 初级生产力的主要限制因子, 后者随降水与潜在蒸 
表 1 陆地生态系统碳储量的估计

Table 1 Estimated range of total carbon storage by ecosystem

\begin{tabular}{|c|c|c|c|c|c|}
\hline \multirow[b]{2}{*}{$\begin{array}{l}\text { 生态系统类型 } \\
\text { Ecosystem type }\end{array}$} & \multirow[b]{2}{*}{$\begin{array}{c}\text { 总土地面积 } \\
\text { Total land area } \\
\left(\times 10^{6} \mathrm{~km}^{2}\right)\end{array}$} & \multicolumn{3}{|c|}{ 全球碳储量 Global carbon storage $(\mathrm{Pg})$} & \multirow{2}{*}{$\begin{array}{c}\text { 碳储量 } \\
(\text { 低 高) } \\
\text { Carbon stored } \\
(\text { Low - High) } \\
\left(\mathrm{kg} \mathrm{hm}^{-2}\right) \\
\end{array}$} \\
\hline & & $\begin{array}{c}\text { 植被 }(\text { 低 高) } \\
\text { Vegetation } \\
\text { (Low - high) }\end{array}$ & $\begin{array}{c}\text { 土壤 (平均) } \\
\text { Soil } \\
\text { (Mean) }\end{array}$ & $\begin{array}{c}\text { 总量 }(\text { 低 高 }) \\
\text { Total } \\
\text { ( Low - high })\end{array}$ & \\
\hline \multicolumn{6}{|l|}{ 森林 Forests } \\
\hline 高纬 High-latitude & 10.3 & $46 \sim 115$ & 266 & $312 \sim 380$ & $303 \sim 370$ \\
\hline 中纬 Mid-latitude & 5.9 & $37 \sim 77$ & 84 & $122 \sim 161$ & $206 \sim 273$ \\
\hline 低纬 Low-latitude & 12.8 & $48 \sim 256$ & 131 & $180 \sim 396$ & $140 \sim 310$ \\
\hline 系统总量 Sub-total & 29.0 & $132 \sim 457$ & 481 & $613 \sim 938$ & $211 \sim 324$ \\
\hline \multicolumn{6}{|l|}{ 草地 Grasslands } \\
\hline 高纬 High-latitude & 10.9 & $14 \sim 48$ & 281 & $295 \sim 329$ & $271 \sim 303$ \\
\hline 中纬 Mid-latitude & 20.1 & $17 \sim 56$ & 140 & $158 \sim 197$ & $79 \sim 98$ \\
\hline 低纬 Low-latitude & 21.7 & $40 \sim 126$ & 158 & $197 \sim 284$ & $91 \sim 131$ \\
\hline 系统总量 Sub-total & 52.6 & $71 \sim 231$ & 579 & $650 \sim 810$ & $123 \sim 154$ \\
\hline \multicolumn{6}{|l|}{ 农业用地 Agroecosystems } \\
\hline 高纬 High-latitude & 3.4 & $8 \sim 18$ & 45 & $52 \sim 62$ & $156 \sim 187$ \\
\hline 中纬 Mid-latitude & 12.7 & $21 \sim 52$ & 134 & $155 \sim 186$ & $122 \sim 147$ \\
\hline 低纬 Low-latitude & 9.5 & $20 \sim 72$ & 85 & $105 \sim 157$ & $110 \sim 164$ \\
\hline 系统总量 Sub-total & 25.6 & $49 \sim 142$ & 264 & $313 \sim 405$ & $122 \sim 159$ \\
\hline \multicolumn{6}{|l|}{ 其它 Others } \\
\hline 高纬 High-latitude & 18.6 & $3 \sim 31$ & 65 & $69 \sim 96$ & $37 \sim 52$ \\
\hline 中纬 Mid-latitude & 11.1 & $9 \sim 25$ & 61 & $70 \sim 86$ & $64 \sim 78$ \\
\hline 低纬 Low-latitude & 8.8 & $4 \sim 16$ & 34 & $38 \sim 50$ & $43 \sim 56$ \\
\hline 系统总量 Sub-total & 38.5 & $16 \sim 72$ & 160 & $177 \sim 232$ & $46 \sim 60$ \\
\hline 总量 Grand total & 145.7 & $268 \sim 901$ & 1484 & $1752 \sim 2385$ & $120 \sim 164$ \\
\hline
\end{tabular}

生态系统类型划分建立在 IGBP 的分类基础上 (Loveland et al . , 2000)。“其它”包括湿地 ,人居地和裸地。总土地面积包括格陵兰和南极。 这里的土壤碳蓄量为 $1484 \mathrm{Pg}$, 不同于前面的 $1555 \mathrm{Pg}$, 是因为纬度带划分所采用的地图与 FAO 用的世界土壤图 (FAO,1991)不同 Land area for each ecosystem is based on the Global Land Cover Characteristics database. The category " other" includes wetlands , human settlements , and barren land. The estimate of $1484 \mathrm{Pg} \mathrm{C}$ in soil is lower than $1548 \mathrm{Pg} \mathrm{C}$ reported by Batjes (1996) , because ecosystems areas were summarized within latitudinal bands from a different resolution map than that used in the FAO soil map of the world(FAO , 1991)

来自 Source : Matthewe et al. ,2000

发之比(降水量/潜在蒸发量)的减小而减小 (James et al . ,2001)。降水量和降水的季节分配与草原、荒 漠、湿地等的净初级生产力直接相关，年均降水量与 年净初级生产力存在显著的线性相关。对农作物而 言, 降水的季节变化(特别是作物生长期间降水的持 续性)比年降水量和潜在蒸发对作物年产量的影响 更大(Donald et al. , 1997)。

\section{2 温室效应对陆地生态系统碳密度的影响}

\section{2 .1 全球变暖}

由于大气中温室气体浓度的增加, 全球气温升 高明显。根据对全球大约 7000 个气象台站的气象 资料所做的分析，有充分的数据表明 :自 19 世纪后 半叶以来, 全球地表年平均气温升高 $0.3 \sim 0.6{ }^{\circ} \mathrm{C}$ (James et al ，2001)。温度升高可以延长生长季, 使 植物有更多时间用于光合, 并将其产物储存到根、木 质部及可收获器官中, 增加植物生物量积累。另一 方面呼吸作用随温度升高呈指数增长趋势, 温度每 升高 $10{ }^{\circ} \mathrm{C}$ 呼吸速率几乎会加倍, 所以假定其它条 件都不变, 升温对呼吸的影响可能使植物用于形态
建成的那部分初级生产量锐减 (Donald et al. , 1997)。暖冬将有可能通过提高年呼吸量使古老的 北方森林由碳汇变为碳源 (Valentini，2000)。高纬 度生态系统中，分解过程可能比生产过程更快地响 应这种气候变化，从而导致系统净碳支出。所以，其 综合结果可能是陆地生态系统碳密度变化微小 (Donald et al. , 1997)。King 等(1997)的模拟研究表 明: 假设 $\mathrm{CO}_{2}$ 浓度不变, 升温将使全球净初级生产力 提高 10\% 14.8\% 但 NPP 的变化几乎被气温升高 随之引发的分解作用加强所抵消, 最终陆地生态系 统碳储量 (Terrestrial ecosystem carbon, TEC) 的变化范 围从增加 $1.1 \%$ 到减少 $1.1 \%$ 。

许多年来,生态系统动态的理论模型认为, 全球 变暖会通过提高生态系统的呼吸作用多于其提高光 合作用来减小陆地碳汇的规模和数量 (Valentini， $2000)$ 。但是这个预测存在着许多问题, 多数观察结 果并不支持这类预测升温会减少土壤碳储量的模 型。例如 Fitter (1999) 在研究温度对植物分解和土 壤碳储库的影响中发现人工加热的高山草地生态系 
统, 其根系生长和死亡的数量基本相当。预测与现 实出现差异的原因在于 : 1) 以往的生态系统模型过 高地估计了土壤呼吸对温度的依赖性 (Liski et al. , 1999)；2) 全球变暖可能会提高某一种物理化学过 程的速率, 而这种过程可以将有机碳转化到更为稳 定的土壤有机物质库中，以保护碳免受分解，该过程 需要一定数量的活化能, 以使有机物质和土壤矿物 结合起来, 减少分解影响, 而高温正好可以提供这种 能量(Thornley \& Cannell , 2001)。所以全球变暖的结 果并不会促使陆地碳汇向大气释放额外的 $\mathrm{CO}_{2}$, 从 而恶化全球升温，事实上，很可能正相反的是温度升 高会引发负反馈, 沉降更多的碳，减缓 $\mathrm{CO}_{2}$ 诱发的全 球变暖趋势 (Liski et al. , 1999 ; Thornley \& Cannell， 2001)。

\subsection{2 $\mathrm{CO}_{2}$ 浓度升高}

$\mathrm{CO}_{2}$ 浓度升高会直接影响植物生长和水分利 用。高浓度 $\mathrm{CO}_{2}$ 的环境有助于植物提高其光合速 率，从而增加其生产力和碳水化合物储量。空气中 $\mathrm{CO}_{2}$ 的增多会提高植物对 $\mathrm{CO}_{2}$ 的可获得性，所以植 物叶片的气孔开放程度将变小, 开放时间也缩短, 植 物蒸腾作用将减弱 (许大全, 2002)。光合增强和蒸 腾抑制共同作用可以提高植物的水分利用效率, 即 消耗单位量的水分可以产生更多的生物量。实验室 研究和园艺实验都表明将 $\mathrm{CO}_{2}$ 浓度增加到工业前水 平的两倍左右 (大约 $600 \mathrm{ppm}$ ) , 就会使 $\mathrm{C}_{3}$ 植物的生 物量提高 $35 \%, \mathrm{C}_{4}$ 植物提高 $10 \% \sim 15 \%$ (Donald et $a l$. , 1997)。此外, $\mathrm{CO}_{2}$ 因施肥效应还可以缓解湿 度、盐分胁迫，可能还有某些养分缺失对作物带来的 伤害 (Idso \& Idso，1994)。 $\mathrm{CO}_{2}$ 的施肥效应增加的陆 地碳汇可达 $(1.0 \pm 0.5) \mathrm{Pg} \mathrm{G} \mathrm{a} \mathrm{a}^{-1}$ (Schimel ,1995)。 但高浓度 $\mathrm{CO}_{2}$ 的环境中生长的植物 $\mathrm{C} / \mathrm{N}$ 较高, 贫营 养, 昆虫需要采食更多生物量来满足其对氮的需求， 同样的，这将在许多方面引出难以预测的变化 (Field et al . , 1992)。实际上, $\mathrm{CO}_{2}$ 的施肥效应带来的生物 量增加不会一直持续下去, 当植物长期处于高 $\mathrm{CO}_{2}$ 浓度时，一些植物的光合速率会逐渐下降, 最终接近 或低于在普通大气 $\mathrm{CO}_{2}$ 浓度下生长的对照水平，即 所谓的光合作用对高浓度 $\mathrm{CO}_{2}$ 的适应 (Dulucia et $a l$. , 1985) ,但其机制尚不是很清楚。

Williams 等 (2000) 在美国堪萨斯州所做的 $\mathrm{CO}_{2}$ 增加对高草草原碳动态影响的实验表明, 空气中的 $\mathrm{CO}_{2}$ 增加会使 $15 \mathrm{~cm}$ 浅层土壤的平均水分含量较之 普通生态系统提高 $15 \%$, 高浓度 $\mathrm{CO}_{2}$ 地段的土壤湿 度与土壤微生物活动正相关，后者比周围环境提高
$14 \%$ 。土壤微生物活动提高会增加碳氮含量。所以 他们认为空气中的 $\mathrm{CO}_{2}$ 提高可以增加高草草原土壤 碳沉降, 推之到地球上的所有温带草地(占陆地面积 的 $10 \%$ ）,下世纪其土壤 $15 \mathrm{~cm}$ 浅层会额外沉降 1.3 $\mathrm{Pg} \mathrm{C}$ 。由此可见, $\mathrm{CO}_{2}$ 浓度上升将在短期内提高植 被和土壤的碳密度。

Pretice 和 Fung (1990)的 2 倍 $\mathrm{CO}_{2}$ 模拟实验也表 明, $\mathrm{CO}_{2}$ 浓度增加引发的全球变暖会使海平面上升， 湿度增加, 植物带的分带发生变化, 如热带雨林和寒 带落叶阔叶林的面积增加, 导致生物库和土壤库的 碳咜量增加, 沙漠、半沙漠寒温带常绿针叶林和冻原 的面积减少, 导致土壤碳库减少, 但其减少量远小于 前两者生物库增加的量。由此可见 2 倍 $\mathrm{CO}_{2}$ 可能最 终增加植物和土壤碳库的碳存储量。King 等 (1997) 的模拟实验也证实, 假设气候不发生变化, $\mathrm{CO}_{2}$ 加倍 会使全球净初级生产力 (NPP) 提高 $16.9 \%$, 使陆地 生态系统碳存储量提高 $14.9 \%$; 若 $\mathrm{CO}_{2}$ 浓度和气候 同时发生变化, NPP 将增加 $30.2 \% \sim 36.5 \%$, 其增 加量多于分解加速带来的抵消量, TEC 将增加约 $15.4 \% \sim 18.2 \%$ 。

$\mathrm{CO}_{2}$ 浓度升高可能会改变碳在生态系统内部各 组分间的分配,Bruce 和 Ellsabeth(1997) 所做的草地 $\mathrm{CO}_{2}$ 加倍实验也表明光合作用加强导致的碳增加要 多于生态系统碳存储量的增加, 大部分增加的碳被 分配到循环较快的地下碳库, 即高浓度 $\mathrm{CO}_{2}$ 导致的 草地碳循环的提高要多于其对碳存储的影响, 这也 解释了许多 $\mathrm{CO}_{2}$ 研究中的不平衡现象。

\section{3 土地利用与覆盖变化 (LUCC)}

土地利用和覆盖的变化直接改变着陆地生态系 统的结构和功能, 以及区域乃至全球范围内的生物 地球化学循环 (Schlesinger, 1997)。从 1850 1995 年, 由于土地利用变化(包括植被砍伐、退化、土壤耕 作、植物残体腐化及弃耕地恢复等) 导致的净碳通量 (由陆地到大气) 由 $397 \mathrm{Pg} \mathrm{C}$ 增加到 $2103 \mathrm{Pg} \mathrm{C}$ (Houghton ,1995)。从 1850 到 1998 年, 全球由于土地 利用变化导致的全球 $\mathrm{CO}_{2}$ 净排放中约 $87 \%$ 来源于 森林砍伐, 约 $13 \%$ 来自中纬度草地开垦成农田 (Houghton et al . 1999)。

\subsection{1 森林生态系统管理}

热带地区大规模的毁林活动对陆地生态系统碳 储量和碳排放速率有极为重要的影响，从 1979 1989 年, 热带郁闭森林年损失面积大约增加了 $90 \%$ (Myers，1991) ,目前所有土地利用与覆盖变化引起 的 $\mathrm{CO}_{2}$ 排放中, 有 $60 \%$ 来自热带国家过去 50 年释放 
的碳。由于原始森林受毁导致的生物量变化必将影 响森林的碳储量。与自然森林相比, 热带地区种植 园森林的地上生物量较自然森林低 $20 \%$ ～50\% ; 温 带地区生产性森林的地上生物量较自然森林低 40\% 50\%（WBGU，1998)。次生森林和不定期砍 伐森林的木本植物生物量较自然森林低 $30 \%$ 80\% (FAO/UNEP , 1981)。因此, 原始森林受剧烈扰 动后形成的次生林的固碳能力将大幅度降低。

森林砍伐还干扰土壤, 使土壤有机质暴露并与 氧气充分接触增高土壤温度和增加降水量从而极 大地促进土壤呼吸作用, 使森林土壤中的大量碳释 放到大气中 (Houghton, 1995)。尽管幼龄林比老龄 林和成熟林具有更高的碳同化速率, 能截留更多的 碳, 但这并不意味着破坏现有的自然林地变为人工 造林更为有利，因为皆伐造成的温度及水分条件的 变化使土壤呼吸消耗在很多年内都超过幼年树木同 化吸收的碳, 即森林欣伐后很多年可能一直都是碳 源( Schlesinger, 1997)。另外，森林收获早也会降低 其固碳能力。平均而言 经过几个 生长-收获” 周期 后, 人工林的碳储量仅是未干扰立地或原始林的 30\% (Cooper , 1983)。

至 1998 年已有约 $750 \times 10^{6} \mathrm{hm}^{2}$ 的森林被用作 农田，占土地利用变化面积的 $45 \%$ （Lal et al.， 1998)。森林用作农田后, 土壤碳储量的损失因气 候、土壤初始碳含量和管理措施的不同而不同。一 般而言，1 m 土层内的土壤碳损失 $25 \%$ ～30\% 耕作 层 $(0 \sim 20 \mathrm{~cm})$ 损失最大, 可达 $40 \%$ 。森林用作农田 后土壤碳含量损失巨大并一直处于下降状态, 一般 需 $20 \sim 50$ 年才可使土壤碳含量增加 (Houghton, 1995)。

\subsection{2 草地管理}

影响草原生态系统碳咜量的土地利用活动包括 草地开圼和过度放牧。由于人口增加带来的粮食需 求压力, 大量草地被开圼成农田。至 1998 年, 全球 已有约 $660 \times 10^{6} \mathrm{hm}^{2}$ 的草地被开圼成农田, 占土地 利用变化面积的近 40\% (Lal et al. , 1998)。草地开 星后伴随的烧荒措施使原来固定在植被中的碳素全 部释放到大气中 ;开圼促进了土壤呼吸作用，加速了 土壤有机质的分解; 多年生牧草被作物取代后使初 级生产固定的碳素向土壤中的分配比例降低，收割 又减少了地上生物量中碳素向土壤的输入 (Anderson \& Coleman , 1985)。草地开圼成农田使得碳储量由 草地的 $116 \mathrm{Mg} \mathrm{G} \mathrm{hm}$-2减少到农田的 $87 \mathrm{~kg} \mathrm{G} \mathrm{hm}^{-2}$, 即碳储量损失了 $19 \mathrm{Pg}\left(28.8 \mathrm{Mg} \mathrm{G} \mathrm{hm}^{-2}\right)$, 这些碳储
量的减少超过了热带森林转换为农田导致的全球碳 损失平均值 $24.5 \mathrm{Mg} \mathrm{G} \mathrm{hm}{ }^{-2}$ (Eswaran et al. ,1993)。 就全球碳平均而言, 草地开圼成农田导致 $1 \mathrm{~m}$ 深度 土层内的土壤碳损失 20\%～30\%（WBGU，1998）, 与森林用作农田后 $1 \mathrm{~m}$ 土层内的土壤碳损失 $25 \%$ $30 \%$ (Houghton，1995) 相当。Houghton 估计 1850 1980 年期间由于开圼导致的草原生态系统碳素净 损失量约为 $10 \mathrm{Pg}$ C。过度放牧使草地初级生产力 降低, 家畜采食减少了碳素由植物调落物向土壤中 的输入, 而且可促进草地土壤的呼吸作用, 从而加速 碳素从土壤向大气的释放。如内蒙古锡林河流域羊 草草原的研究结果表明, 40 年来过度放牧使表层土 壤( $0 \sim 20 \mathrm{~cm}$ ) 中碳的财存量降低了 $12.4 \%$ ( Li \& Chen, 1997)。

土地利用的变化不一定总是造成碳密度的降 低, 南美洲管理完善的牧场可以具极高的碳密度, 尤 其是扎根很深的情况 (Fisher et al. , 1994)。欧洲许 多地区的大面积弃耕农地正逐渐恢复成森林植被， 我国正在实施的大面积人工造林, 退耕还林还草也 是提高生态系统碳汇能力 增加碳密度的有力措施。 森林重建和再造可以提高碳沉降速率, 使北方地区 地上地下生物量增加 $0.4 \sim 1.2 \mathrm{Mg} \mathrm{hm}^{-2} \mathrm{a}^{-1}$, 温带 地区增加 1.5 4.5 $\mathrm{Mg} \mathrm{hm}^{-2} \mathrm{a}^{-1}$ 热带地区可增加 $4 \sim 8 \mathrm{Mg} \mathrm{hm}^{-2} \mathrm{a}^{-1}$ (Dixon et al. , 1994)。

\section{4 土壤养分}

北方中纬度地区的森林是一个巨大的陆地碳 汇 如果不考虑养分限制, $\mathrm{CO}_{2}$ 的施肥效应带来的碳 沉降大幅度增加在这些森林中是完全可能的。但森 林一般属于中营养或贫营养的地区, 树木的生长常 常受到养分供应的限制, 特别是氮的限制。认为森 林碳沉降的增加可能会局部抵消大气 $\mathrm{CO}_{2}$ 的增多， 现在证明是过于乐观了。在两个 $\mathrm{CO}_{2}$ 浓度较高的成 熟松林中所做的实验表明，在一个贫营养地区，如果 没有额外的养分添加, $\mathrm{CO}_{2}$ 升高带来的生物量增长 及碳储量增加都是无法观测到的 ( Fan et al. , 1998)。高 $\mathrm{CO}_{2}$ 的施肥效应需要通过添加养分来表 现。

在一定范围内施氮, 可以增加作物叶面积, 改善 和提高光合作用 ( Schlesinger, 1997)。黄建晔等 (2002) 对水稻进行的开放式空气 $\mathrm{CO}_{2}$ 浓度增高 (FACE) 实验也表明,FACE 处理可以显著提高水稻 产量, 即碳储量会增加, 而这种增产在高 $\mathrm{N}$ 条件下 更明显。提高农业生产力通过提高碳向土壤的输入 也能增加土壤碳咜存(刘允芬, 1999)，所以农业生产 
大量施用有机肥和绿肥, 会使植物碳库和土壤有机 碳库都有较大增加。在同等条件下施肥, 瘦薄红壤 中有机碳会较快积累, 也即通过对瘦薄红壤的改良， 可以在其中固持较多的大气 $\mathrm{CO}_{2}$ (李忠佩等,2002)。

\section{5 生物多样性}

植物多样性及其组分会影响由于大气 $\mathrm{CO}_{2}$ 浓度 提高和 $\mathrm{N}$ 沉积增加带来的生物量增长及生态系统 碳储量的增多。Reich 和 Knops (2001) 通过模拟实 验验证发现，在贫物种地区， $\mathrm{CO}_{2}$ 或 $\mathrm{N}$ 浓度的提高， 或二者共同提高带来的生物量积累要小于多物种集 合区。

多样性的减少不仅造成植物种类的损失, 而且 会导致其作为碳汇能力的降低。英国 Silwood Park 皇家学院种群生态学研究中心的科学家们通过实验 证实生物多样性锐减会改变生态系统在生物量生 成, 营养成分保持和分解, 以及 $\mathrm{CO}_{2}$ 吸收方面的能 力。高多样性种群消耗从大气中吸收的 $\mathrm{CO}_{2}$ 远多于 低多样性种群。一般情况下, 植物多样性的减少意 味着生态系统固定 $\mathrm{CO}_{2}$ 的能力降低, 植物多样性越 少，碳存储量越少(Shahid et al，，1994）。

外来种引入也会影响碳存储 (Donald et al. , 1997)。Regina 大学的两位科学家证实, 由北亚引入 的一种有冠毛的冰草 (Agropyron cristatum) , 其下的 土壤含碳、含氮量要远远小于本地生冰草, 这可以用 它们各自的生长对策来解释。有冠毛冰草的地上部 分分支多, 地下根系较浅, 而本地种地上部分较矮, 但根系统庞大。由此推论外来种可能会使碳储库变 小, 但这一方面的研究仍很欠缺, 其影响可能不单纯 是取代本地种, 减少多样性, 改变碳库选择和草地生 态系统的能物流。PAGE 的研究也指出, 一些有毒 草种会降低总体的土壤碳储量, 因为它们储存有机 质的地下根系不如本地种广大(Robin et al .,2000)。

\section{3 展 望}

现在对于碳密度的研究仍存在许多不确定性和 争论之处 (Suzi et al . ,2001), 许多研究选取的地点 及方法是否具有代表性仍值得置疑, 还有一些影响 碳沉降的因子及机制尚不是很清楚 (Schimel, 1995 ）,包括对不同地区的碳密度估算采用的特定模 型是否准确有效也未有定论，因而建立一套统一而 规范的碳密度度量与观测方法以减小数据的不确定 性(Melillo et al . , 1996)，发展针对特定地区特定时 段的碳密度估算模型以减少对过程模拟的不确定性 (Haxeltine et al. , 1996) 是极为必要的。在土壤碳蓄
积量估算、土地利用和覆盖变化，及碳密度估算建模 等方面还存在许多需要深入研究的问题, 碳密度研 究的空间还应该继续拓宽外延, 加强其与碳循环和 碳通量等其它研究的连接。

\section{1 土壤碳蓄积量的不确定性}

土壤圈由于具有巨大的有机碳库, 又是对全球 变化反应灵敏、复杂的系统, 在减少碳汇不确定性方 面理应具有极为重要的作用, 但目前尚未引起足够 重视(方精云等 2001)。土壤分类系统的不统一,采 样方法的差异, 以及选用不同的土壤碳蓄积量计算 方法和参数估计方法使目前的土壤碳蓄积量的估算 存在极大的不一致, 土壤实测数据不充分和缺乏连 续、可靠、完整、统一的土壤剖面数据也使碳密度量 测的可行性大打折扣(王绍强等,2000)。土壤碳氮 含量、质地、容重、根量等理化性质存在很大的空间 差异，气候、母岩、植被和土地利用对土壤碳库容量 的综合影响也很难确定（王绍强等，2003），各地区土 壤厚度和面积统计资料来源不同也是不确定性存在 的重要原因之一（王绍强等, 1999a;1999b)。不同尺 度上的影响因子及主要控制因子也存在很大差异, 所以由此得到的土壤碳蓄积的机理过程模拟及其潜 在分解、固定和储存能力的分析都会有所区别(王绍 强等, 2003)。针对以上问题, 在今后的研究中应着 力于建立适用于全球或区域等不同尺度上的的多个 土壤分类系统, 推进观测采样和度量计算方法的规 范化完善化(方精云等,1996a；倪健，2001），扩充研 究地点 积累更为连续完整的土壤调查数据 (Fang $e t$ $a l$, 1998) 做好不同时空尺度上数据的整合和集成 工作，考虑多方面影响因子的综合作用，提高对土壤 碳密度估测的准确性。

土壤内部碳循环的过程难以观测, 土壤呼吸及 有机质转化过程的不能确定, 也为土壤碳累积过程 和机理研究带来了极大困难。土壤有机碳输入速率 很难定量, 特别是对自然生态系统和范围较小的农 业生态系统。通过调落物或植物残留物的碳输入或 许可以直接测得, 但通过根和根瘤菌的输入很难测 算(Post et al . , 1982)。目前这一方面的研究重点在 于碳从植物到土壤有机质的转化, 土壤有机质中的 碳沉降速率, 土壤有机质矿质化及环境因子影响下 的养分供应对其的影响。使用同位素技术跟踪土壤 碳循环现在仍是解决这一问题的主要途径。

\section{2 碳存储模型的建立与尺度问题}

基于对碳存储过程限定性的不同理解和各个影 响因子的不同参数化条件, 每一个模型的建模机理 
和参数选用都存在着差异, 因此各模型的适用范围 和有效性也各不相同 (Cramer et al .,1999)。由于陆 地生态系统的类型、性质、分布面积，及其所处的气 候土壤等自然地理特点, 以及它们对气候变化的反 应特性都存在较大的空间差异性, 还有气候的年波 动, 偶发事件(如森林火灾和大面积病虫害)的出现 等年际及季节变化的难以预测 (方精云等,2001) , 使得任何一个模型都不能胜任所有时空尺度上的碳 储量计算, 每个模型的估算及预测能力都存在不足 和缺陷，因而在特定地区完善和改良现有的用于模 拟分析碳储量的各类模型, 提高参数的易获取性和 有效性，减少模拟结果的不确定性 增加模型间的可 比性是目前的建模工作亟待解决的问题。

对于复杂尺度上碳存储的建模, 鉴别那些易于 测量和从当地数据源易于获取的变量, 以及可以预 测碳密度的变量是极为关键的, 这些数据要在统计 分析和模型模拟中都可以使用, 以获得碳存储量估 测的时空整合 (Cramer et al . , 1999)。选用的变量必 须在待研究尺度上可测, 如果在指定尺度上难于测 定，即使它可以提高模型的适用性也要将其去除。 合适的预测变量的选用可以从对碳循环过程的了解 中获得指导。尽管大尺度模型一般不必包括细节性 机理，但是当碳密度的控制因素非线性地作用时，多 精度水平的估测极为重要, 过分简化会导致很严重 的错误 (Thomas et al. , 1988)。所以在利用模型模 拟陆地生态系统碳储存过程及潜力的研究中, 我们 要尽量开展多尺度的调查研究, 将小尺度研究外推 到较大空间领域增加模型的适用性和准确性。

\section{3 土地利用及覆盖变化}

土地利用及覆盖变化 ( LUCC) 是影响陆地生态 系统碳密度格局的一个重要因子, 但其难于监测的 特性给碳存储过程研究和数量估算带来了极大的困 难 ,Houghton(1996)认为许多碳储量及碳循环研究的 不确定性是由于缺乏世界森林植被毁林和重建速率 的全面数据造成的。运用卫星影像等遥感手段监测 不同时空尺度上的土地利用及覆盖的变化, 具有重 要而独特的价值。在碳储量的研究中, $3 \mathrm{~S}$ 技术与机 理分析，模型建立的结合仍是一个重要的方面。

土地利用与覆盖变化可以综合反映一个地区的 社会经济条件及人为决策因素之间的关系, 只有更 好地揭示土地利用与覆盖变化对陆地生态系统碳密 度的影响机理, 才能真实地探究碳密度分布规律, 预 测其储量的变化 (Suzi et al. ,2001)。针对土地利用 与覆盖变化影响碳储量的机制以及 LUCC 建模的研
究还有很大的空间。目前的研究重点应该是:分析 土地利用及覆盖变化的主要社会经济驱动力和自然 驱动力, 以及它对这些驱动力的反馈机制, 充分考虑 土地利用和覆盖变化对政策、技术进步、人口增长、 经济发展、市场变化等社会变量的灵敏性(李晓兵， 1999)，并将其加入陆地生态系统碳密度估测模型 中 建立融自然、经济、社会状况为一体的诊断决策 模型。

\section{参 考 文 献}

Anderson, D. W . \& D. C. Coleman. 1985. The dynamics of organic matter in grassland soils. Journal of Soil and Water Conservation, 40: $211 \sim 216$.

Batjes, N.H. 1996. Total carbon and nitrogen in the soils of the world. European Journal of Soil Science, 47: 151 163 .

Bohn, H.L.1976. Estimate of organic carbon in world soils. Journal of Soil Science Society of America, 40:468 470.

Bruce, A. H. \& A. H. Ellsabeth. 1997. The fate of carbon in grasslands under carbon dioxide enrichment. Nature, 388: 576 $\sim 580$.

Christian, P. G. \& G. R. Michael. 2000. Evidence that decomposition rates of organic carbon in mineral soil do not vary with temperature. Nature, 404: $858 \sim 860$.

Cooper, C. F. 1983. Carbon storage in managed forests. Canadian Journal of Forest Research, 13: $14 \sim 55$.

Cramer, W., D. W. Kicklighter \& A. Bondeau. 1999. Comparing global models of terrestrial net primary productivity $(N P P)$ : overview and key results. Global Change Biology, 5(1):1 15 .

Dixon, R., J. Winjum, K. Andrasko, J. Lee \& P. Schroeder. 1994. Integrated systems: assessment of promising agroforest and alternative land-use practices to enhance carbon conservation and sequestration. Climatic Change, 30: 1 23 .

Donald, J. W., J. Y. W. Barbara \& R. Norm. 1997. Global climate change and the world food supply. Prepared for The Illinois World Food and Sustainable Agriculture Program Conference. $30 \sim 39$.

Dulucia, E.H. , T. W. Sasek \& B.R. Strain. 1985. Photosynthetic inhibition after long-term exposure to elevated levels of atomospheric carbon dioxide. Photosynthesis Research, 7:175 184 .

Emily, M., R. Payen, M. Rohweder \& S. Murray. 2000. Pilot analysis of global ecosystem: forest ecosystems. World Resource Institute.

Eric, A.D., S. Trumbore \& R. Amundson. 2000. Soil warming and organic carbon content. Nature, 408: $789 \sim 790$.

Eswaran, H. , E. Vandenberg \& P. Reich. 1993. Organic carbon in soils of the world. Journal of Soil Science Society of America, 57: $192 \sim 194$.

Fan, S., M. Gloor, J. Mahlman, S. Pacala, J. Sarmiento, T. Takahashi \& P. Tan. 1998. A large terrestrial carbon sink in North America implied by atmospheric and oceanic carbon dioxide data and models. Science, 282: $442 \sim 446$.

Fang, J. Y., S. H. Liu \& Q. Zhao. 1998. Factors affecting soil respiration in reference with temperature' $s$ role in global scale. Chinese Geographical Science (中国地理科学), 8: 246 255 .

Fang, J. Y. (方精云)，G.H. Liu(刘国华) \& S. L. Xu(徐嵩 龄). 1996a. Carbon reservoir of terrestrial ecosystem in China. In: Wang, G.C. (王庚辰) \& Y. P. Wen (温玉璞) eds. Monitoring and relevant process of green house gas concentration 
and emission. Beijing: China Environmental Science Press. 109 $\sim$ 128. (in Chinese)

Fang, J.Y. (方精云) , G.H. Liu(刘国华) \& S. L. Xu (徐嵩 龄). 1996b. Carbon pools in terrestrial ecosystems in China. In: Wang, R.S. (王如荪), J.Y. Fang(方精云), L. Gao(高 林) \& Z.W. Feng(冯宗炜) eds. Hot spots in modern ecology. Beijing: Chinese Science and Technology Press. 251 277. (in Chinese)

Fang, J.Y. (方精云)，S. L. Piao(朴世龙) \& S. Q. Zhao (赵 淑清). 2001. The carbon sink: the role of the middle and high latitudes terrestrial ecosystems in the northern hemisphere. Acta Phytoecologica Sinica(植物生态学报), 25:594 602 (in Chinese with English abstract)

FAO. 1991. The digitized soil map of the world. World soil resources report 67 on CD-ROM. Rome: FAO.

FAO/UNEP (Food and Agriculture Organization of the United Nations/United Nations Environment Programme). 1981. Tropical forest resources assessment project. Rome, Italy: FAO. $22 \sim 53$.

Field, C. B., F.S. Chapin III, P. A. Matson \& H. A. Mooney. 1992. Response of terrestrial ecosystems to the changing atmosphere: a Resource-based Approach. Annual Review of Ecology and Systematics, 23: $201 \sim 235$.

Fisher, M. J., I. M. Rao, M. A. Ayarza, C. E. Lascano, J. I. Sanz, R.J. Thomas \& R.R. Vera. 1994. Carbon storage by introduced deeply rooted grasses in the South American savannas. Nature, 371: $236 \sim 238$.

Fitter, A. H., G. K. Self, T. K. Brown, D. S. Bogie, J. D. Graves, D. Benham \& P. Ineson. 1999. Root production and turnover in an upland grassland subjected to artificial soil warming respond to radiation flux and nutrients, not temperature. Oecologia, 120: $575 \sim 581$.

Food and Agriculture Organization of the United Nations (FAO). 1995. Digital soil map of the world (DSMW) and derived soil properties on CD-ROM. Rome: UNESCO.

Gaston, K. T. B., T. Blackburn \& J. Spicer. 1998. Reply from K. J. Gaston, T. M. Blackburn and J. I. Spicer. Trends in Ecology and Evolution, 13:242 253.

Haxeltine, A., I. C. Prentice \& I.D. Creswell. 1996. A coupled carbon and water flux model to predict vegetation structure. Journal of Vegetation Science, 7: 651 656 .

Houghton, R.A. 1995. Changes in the storage of terrestrial carbon since 1850. in: Lai, R. ed. Soils and global change. Florida: CRC Press, Inc. Boca Raton. 45 65.

Houghton, R.A. 1996. Land-use change and the temporal record. In: Apps, M.J. \& D.T. Price eds. Forest management and the global carbon cycle. Berlin: Springer-Verlag. 117 134 .

Houghton, R. A., J. L. Hackler \& K. T. Lawrence. 1999. The U.S. carbon budget: contributions from land use change. Science, 285:574 578.

Huang, J. Y. (黄建晔), H.J. Yang(杨洪建), G.C. Dong(董 桂春), Y.L. Wang(王余龙), L.X Yang(杨连新) \& Y.H. Shan(单玉华). 2002. Effects of free air $\mathrm{CO}_{2}$ enrichment (FACE) on yield formation in rice (Oryza sativa) . Chinese Journal of Applied Ecology (应用生态学报), 13:1210 1214. (in Chinese with English abstract)

Idso, K. E. \& S. B. Idso. 1994. Plant responses to atmospheric $\mathrm{CO}_{2}$ enrichment in the face of environmental constraints: a review of the past 10 year's research. Agricultural and Forest Meteorology, 69:153 203.

James, J.M., O.F. Canziani, N. A. Leary, D. J. Dokken \& K. S. White. 2001. Climate change 2001: impacts, adaptation and vulnerability. Cambridge: Cambridge University Press. $31 \sim 36$. King, A. W., M. W. Post \& D. S. Wullschleger. 1997. The potential research of terrestrial carbon storage to changes in climate and atmospheric $\mathrm{CO}_{2}$. Climatic Changes, 35: 199 227.

Lal, R., J. M. Kimble, R.F. Follett \& C. V. Cole. 1998. The potential of U.S. cropland to sequestrate carbon and mitigate the greenhouse effect. Michigan: Ann Arbor Press. 128 132 .

Li, L. \& Z. Chen. 1997. Changes in soil carbon storage due to over-grazing in Leymus chinensis steppe in the Xilin river basin of Inner Mongolia. Journal of Environmental Science, 9:486 490.

Li, X. B. (李晓兵). 1999. International research on environmental consequence of land use/cover change. Advance in Earth Science (地球科学进展), 14:395 402. (in Chinese with English abstract)

Li, Z.P. (李忠佩) \& X.X. Lin(林心雄). 2002. Characteristics of organic materials decomposition in infertile red soils. Acta Ecologica Sinica(生态学报), 22:1224 1230. (in Chinese with English abstract)

Liski, J., H. Ilvesniemi, A. Makela \& C. J. Westman. 1999. $\mathrm{CO}_{2}$ emissions from soil in response to climatic warming are overestimated-The decomposition of old soil organic matter is tolerant of temperature. America Biology, 28: $171 \sim 174$.

Liu, Y.F. (刘允芬). 1999. Carbon sink functions of Chinese agriculture ecosystem. Agro-environmental Protection(农业环境 保护), 17:197 202. (in Chinese with English abstract)

Liu. G.H. (刘国华), B.J. Fu(傅伯杰), G. Wu(吴钢) \& G. L. Duan(段桂兰). 2003. Soil organic carbon pool and its spatial distribution pattern in the circum Bohai Region. Chinese Journal of Applied Ecology (应用生态学报)，14：1489 1493. (in Chinese with English abstract)

Loveland, T. R., B. C. Reed, J. F. Brown, D. O. Ohlen, Z. Zhu, L. Yang \& J. Merchant. 2000. Development of a global land cover characteristics database and IGBP discover from 1-km AVHRR data. International Journal of Remote Sensing, 21: 1303 $\sim 1330$

Matthews, E., R. Payen, M. Rohweder \& S. Murray. 2000. Pilot analysis of global ecosystem: forest ecosystems. Washington, DC: World Resource Institute. 55 59 .

Melillo, J. M., D. O. Hall \& G. I. Agren. 1996. Executive summary. In: Breymeyer, A. J., D. O. Hall, J. M. Melillo \& G. I. Agren eds. SCOPE - 56: Global change: effects on coniferous forest and grassland. Chichester: Wiley. $10 \sim 16$.

Myers, N. 1991. Tropical forests: present status and future outlook. Climatic Change, 19: $3 \sim 36$.

Ni, J. 2001. Carbon storage in terrestrial ecosystem of China: estimates at different spatial resolutions and their responses to climate change. Climatic Change, 49: $339 \sim 358$.

Olson, J.S. , J. A. Watts \& L. J. Allison. 1983. Carbon in live vegetation of major world ecosystems. Oak Ridge: Oak Ridge National Laboratory.

Post, W. M., A. W. King \& S. D. Wullschleger. 1997. Historical variations in terrestrial biospheric carbon storage. Global Biogeochemical Cycles, 11(1): 99 109 .

Post, W. M., W.R. Emanuel, P.J. Zinke \& A.G. Stangenberger. 1982. Soil carbon pools and world life zones. Nature, 298: $156 \sim 159$.

Prentice, K. C. \& I. Y. Fung. 1990. The sensitivity of terrestrial carbon storage to climate change. Nature, 336: $48 \sim 51$.

Reich, P. B. \& J. Knops. 2001. Plant diversity enhances ecosystem responses to elevated $\mathrm{CO}_{2}$ and nitrogen deposition. Nature, 410: $809 \sim 813$. 
Robin, W., S. Murray \& M. Rohweder. 2000. Pilot analysis of global ecosystem: grassland ecosystems. Washington, DC: World Resource Institute. $49 \sim 53$.

Schimel, D.S. 1995. Terrestrial ecosystem and the carbon cycle. Global Change Biology, 1: $77 \sim 81$.

Schlesinger, W. H. 1997. Biogeochemistry: an analysis of global change. 2nd ed. San Diego, California: Academic Press.

Shahid, N., L. J. Thompson, S. P. Lawler, J. H. Lawton \& R. M. Woodfin. 1994. Declining biodiversity can alter the performance of ecosystems. Nature, 368: $734 \sim 736$.

Suzi, K., S. G. Liu, R.F. Hughes \& A. Pfaff. 2001 . Carbon dynamics, land use-and choie: building a regional scale multidisciplinary model. Motu: Journal of Environmental Management, 69 (1) : $25 \sim 37$.

Thomas, R., G. W. Robert \& G. R. Paul. 1988. SCOPE 35: scales and global change: spatial and temporal variability in biospheric and geospheric process. Scientific Committee on Problems of the Environment (SCOPE), 186 203.

Thornley, J.H.M. \& M.G.R. Cannell. 2001. Soil carbon storage response to temperature: an hypothesis. Annals of Botany, 87: $591 \sim 598$.

Valentini, R. 2000. Respiration as the main determination of carbon balance in European forest. Nature, 401: $861 \sim 864$.

Wang, X.K. (王效科)，Z.W. Feng (冯宗炜) \& Z. Y. Ouyang (欧阳志云). 2001. Vegetation carbon storage and density of forest ecosystems in China. Chinese Journal of Applied Ecology (应用生态学报), 12: 13 16. (in Chinese with English abstract)

Wang, S.Q. (王绍强) \& C.H. Zhou (周成虎). 1999a. Esti- mating soil carbon reservoir of terrestrial ecosystem in China. Geographical Research(地理研究), 18:349 358. (in Chinese with English abstract)

Wang, S.Q. (王绍强)，C.H. Zhou (周成虎) \& C. W. Luo (罗承文).1999b. Studying carbon storage spatial distribution of terrestrial natural vegetation in China. Progress in Geography (地 理科学进展), 18:238 245. (in Chinese with English abstract)

Wang, S.Q. (王绍强), C.H. Zhou (周成虎), K.R. Li (李克 让), S.L. Zhu(朱松丽) \& F. H. Huang (黄方红). 2000. Analysis on spatial distribution characteristics of soil organic carbon reservoir in China. Acta Geographica Sinica(地理学报), 55: $533 \sim 545$. (in Chinese with English abstract)

Wang, S.Q. (王绍强), J.Y. Liu (刘纪远) \& G.R. Yu(于贵 瑞). 2003. Error analysis of estimating terrestrial soil organic carbon storage in China. Chinese Journal of Applied Ecology (应 用生态学报), 14: 797 802. (in Chinese with English abstract)

WBGU. 1998. The accounting of biological sinks and sources under the Kyoto Protocol - a step forwards or backwards for global environmental protection. Bremerhaven: WBGU (German advisory council on global change). $46 \sim 50$.

Williams, M. A., C. W. Rice \& C.E. Owensby. 2000. Carbon dynamics and microbial activity in tallgrass prairie exposed to elevated $\mathrm{CO}_{2}$ for 8 years. Plant and Soil, 227: $127 \sim 137$.

Xu, D. Q. (许大全) . 2002. Photosynthetic efficiency. Shanghai: Shanghai Science and Technology Press. (in Chinese)

Zhang, J.T. (张金屯) \& S. Q. Li(李素清). 2003. Applied ecology. Beijing: Science Press. $534 \sim 539$. (in Chinese)

责任编委 : 欧阳 华 责任编辑 张丽赫 